\title{
Surgery in patients with Ebola virus disease
}

Early-released on Oct. 29, 2014; revised Nov. 11, 2014. Send comments to cjs@ cma.ca.

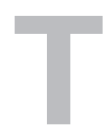
hirty years ago, surgical trainees like me were asked to undertake diagnostic lymph node excisional biopsies in patients with AIDS. Our teachers believed the procedures to be futile and risky. We thought we were invincible. We arranged the set-up so that we could operate alone in double masks, gowns and gloves. The purpose of the inner layer was to permit safer removal of the outer barrier. Similarly we double-bagged laundry and waste.

Yet again, we are faced with a fluid-borne virus whose potential to harm is unknown. A large experience is making clear the steps that should be taken to fight Ebola virus disease (EVD) in West Africa. We have only a tiny experience upon which to base care of patients with EVD in developed countries, such as Canada. Initial hopes that life-supporting procedures, such as mechanical ventilation and hemodialysis, would permit recovery from the advanced stages of EVD are now less certain. Our faith in conventional barrier protocols has been shaken. In this article, I try to address the role of surgery in the care of patients with EVD.

Protocols for the initial care of patients with suspected or confirmed EVD have been developed and practised by Canadian hospitals with the help of the provinces and the Public Health Agency of Canada. ${ }^{1}$ Dr. Robert Fowler has described EVD as a febrile gastrointestinal disease whose lethal effect on fluid and electrolyte homeostasis can be ameliorated through modern critical care. ${ }^{2}$ At the time of writing, 4 patients are believed to have received mechanical ventilation and dialysis for advanced EVD. Two have survived to clear the virus. This success will inspire us to continue to support patients with EVD-induced renal and respiratory failure. Surgeons are commonly asked to consult on other critically ill patients with similar problems. Therefore, we have to face the dilemma of considering surgery in patients with EVD. Information in this area is rapidly accumulating, and clinical care teams will make their own valid decisions on a case-by-case basis. The American College of Surgeons has adapted the Centers for Disease Control and Prevention guidelines for the conduct of surgery in patients with suspected EVD. ${ }^{3}$ Surgeons should consult these guidelines frequently because new information is to be expected. The guidelines are currently silent on who should receive surgery.

When considering any invasive procedure or operation in a patient with EVD, the caregiving team needs to undertake a documented utility-risk analysis, which includes not only the perspective of the patient, but also the $360^{\circ}$ environment. Other modalities of care and the possibility of procedure postponement must be considered, for now, preferable options. The well-accepted preference for methods of rehydration should rigidly favour oral over enteral tube and peripheral over central venous routes of administration. Blood work will have to be minimized and possibly restricted to point-of-care testing. The use of imaging in patients with EVD will also be considerably restricted in comparison to patients without the disease. It will be very difficult to justify the use of arterial lines. Automated noninvasive blood pressure and oxygen saturation monitoring will reduce direct patient contact.

The biggest dilemma for surgeons will be trying to determine futility in a disease with which we have almost no direct experience. The development of organ failure renders the prognosis bleak for patients in health care systems with limited resources, such as those in areas where the outbreaks have occurred. In the developed world, the prognosis is grave with the onset of organ failure, particularly if this occurs after restoration of the fluid, electrolyte and acidbase balance. Liver failure and necrosis have been observed with EVD; failure of supportive measures renders the prognosis hopeless, and neither transplantation nor liver assist devices should be considered. Surgery for peritonitis, gastrointestinal hemorrhage, intestinal perforation or intestinal ischemia is likely to fail. Ebola virus disease may result in anasarca with abdominal compartment syndrome for which mechanical ventilation, complete muscle relaxation and dialysis is preferable to laparotomy. Cardiopulmonary resuscitation is not appropriate for end-stage EVD. Unfortunately experience in Africa has shown that pregnant women with EVD appear to be at an increased risk for spontaneous abortion and pregnancy-associated hemorrhage. Neonates born to mothers with EVD have not survived. ${ }^{4}$

Endoscopy (bronchial or gastrointestinal) is a very highrisk procedure in patients with EVD because of forceful aerosol generation and operator proximity. Diagnostic endoscopy will probably fail a utility-risk analysis, as will most procedures with a therapeutic intent. The added risks related to aerosol generation have to be considered if the laparoscopic route is used for chest or abdominal surgery. Likewise, the use of external drains should be limited because of the risks related to extracorporeal body fluids.

Mechanical ventilation may result in requirements for surgery: tracheostomy should be postponed until the 
patient has cleared the virus; extracorporeal membrane oxygenation would probably not pass a utility-risk analysis; and tube thoracostomy should be considered an aerosol-generating procedure just like endotracheal intubation. The risk of aerosol generation should be mitigated by using properly fitted N95 masks and by removing all nonessential individuals from the room. The surrounding area will require decontamination following the procedure.

Conventional barrier protocols are being strengthened to combat the transmission of Ebola virus. Elements of military protocols for chemical, biological, radiological and nuclear defense may be useful. Gowns should include a hood and boot covers in a one-piece suit. Buddy checks and assistance will reduce failures during the donning and doffing of personal protective equipment. Decontamination with wipes before removal of barriers prevents inadvertent spread. In Africa, reusable gowns with final showers using diluted bleach are preferred by the heroic teams working to contain the outbreak. In the developed world, it is essential that we do not confuse barrier precautions used for diarrhea-causing bacteria, such as Clostridium difficile, with protocols required for EVD. Sterile surgical gowns and gloves may have to be added to EVD barrier suits, which are not sterile.

Unlike in the early days of AIDS, trainees and young surgeons with children should not be asked to operate on patients with EVD. This is a task for experienced surgeons using the smallest possible team in the room. Surgeons asked to consult on patients with EVD should not hesitate to seek advice from surgeons in experienced centres. Fol- lowing the initial fear regarding AIDS, we quickly came to understand and treat HIV. Like many surgeons, I went on to perform the full range of operations, including liver transplantation, on patients infected with HIV. There are good reasons to hope that EVD will likewise be attenuated so that the full range of modern critical care and surgical procedures become possible in patients infected with Ebola virus.

\section{Vivian McAlister, MD}

Coeditor, Canadian Fournal of Surgery

Competing interests: None declared.

DOI: $10.1503 /$ cjs. 015514

\section{References:}

1. Canadian Critical Care Society, Canadian Association of Emergency Physicians, Association of Medical Microbiology \& Infectious Diseases Canada. Ebola clinical care guidelines: a guide for clinicians in Canada. Interim Report, August 29, 2014. Available: www.ammi.ca /media/69846/Ebola\%20Clinical\%20Care\%20Guidelines\%202\%20 Sep\%202014.pdf (accessed 2014 Oct. 29).

2. Fowler RA, Fletcher T, Fischer WA II, et al. Caring for critically ill patients with Ebola virus disease. Perspectives from west Africa. A $m \mathcal{F}$ Respir Crit Care Med 2014;190:733-7.

3. Wren SM, Kushner AL. Surgical protocol for possible or confirmed Ebola cases. American College of Surgeons 7 October 2014. Available: www.facs.org/ebola/surgical-protocol (accessed 2014 Oct. 29).

4. Jamieson DJ, Uyeki TM, Callaghan WM, et al. What obstetriciangynecologists should know about Ebola: a perspective from the Centers for Disease Control and Prevention. [Epub ahead of print]. Obstet Gynecol 2014. 